\title{
Efeito da pressão osmótica no processamento e avaliação da vida de prateleira de tomate seco
}

\section{Effect of osmotic pressure in the processing and evaluation of the shelf life of dried tomato}

\author{
Virlane Kelly Lima da Silva ${ }^{1}$; Érika Sousa Pinheiro'; Maria Aliciane Fontenele \\ Domingues ${ }^{1}$; Andréa Cardoso de Aquino ${ }^{1}$; Evânia Altino Figueiredo ${ }^{2}$; \\ José Maria Correia da Costa ${ }^{2}$; Patrícia Beltrão Lessa Constant ${ }^{2 *}$
}

\section{Resumo}

Após a colheita, o tomate (Lycopersicon esculentum Mill) apresenta-se como um fruto altamente perecível, tendo um curto período de vida para utilização adequada, sendo a sua industrialização uma importante forma de conseguir um melhor e mais amplo aproveitamento diminuindo o percentual de perdas. Este trabalho teve como objetivo, avaliar a influência do processo de desidratação osmótica seguida de secagem por convecção forçada em secador de cabine, nas características físico-químicas, microbiológicas e sensoriais do tomate. Foram testadas separadamente duas soluções xaropes: $\mathrm{NaCl}$ a $10 \%$ e $\mathrm{NaCl} 5 \%$ + sacarose $10 \%$. Após desidratação, acondicionamento a quente com condimento a base de óleo, azeite e orégano e pasteurização os produtos foram armazenados a temperatura ambiente e em ausência de luz. Nos tempos 0, 15, 30 e 45 dias foram feitas análises físico-químicas, microbiologias e sensórias. O emprego de desidratação osmótica partindo-se de solutos e concentrações distintas influenciou de forma diferenciada nas características físico-químicas e sensoriais do tomate seco ao longo do armazenamento. A utilização da mistura $\mathrm{NaCl}$ e sacarose apresentou a melhores resultados no que diz respeito a aceitação pelo consumidor. Com relação ao tempo de armazenamento, não foi constatada diferenças nas avaliações sensoriais, indicando que embora tenham ocorrido alterações físico-químicas, elas não foram significativamente percebidas pelos provadores.

Palavras-chave: Secagem, tomate, osmodesidratação

\begin{abstract}
After harvest, the tomato (Lycopersicon esculentum Mill) is a fruit highly perishable with a short period of life for appropriate use, and its industrialization an important way to achieve a better and wider use by reducing the percentage of losses. The objective of the present study was to evaluate the influence of the dehydrated osmotic process dried by convection forced in booth dryer, on the physical-chemistry microbiologic and sensorial characteristics of the tomato. Tests were done with $\mathrm{NaCl} 10 \%$ (treatment A) and $\mathrm{NaCl} 5 \%+10 \%$ (treatment B). After dehydrated, storing hot with oil flavouring, olive oil and oregano and pasteurized, wares have been stored at environment temperature and on absence of light. At $0,15,30$ and 45 days analysis physical-chemistry, microbiologist and sensorial have been realized. It was possible to certify that the use of dehydrated osmotic breaking with solutes and different concentrations
\end{abstract}

\footnotetext{
1 Mestrandas em Ciência e Tecnologia de Alimentos, UFC. Universidade Federal do Ceará - Depto. de Tecnologia de Alimentos; Avenida Mister Hull s/n - Campus do Pici - Bloco 848 - Caixa Postal 12.140 Cep 60021970 - Fortaleza - CE. E-mail: virlanekelly@yahoo.com.br; erika_mestrado@yahoo.com.br; aliciane_fontenele@yahoo.com.br; dea.lara@terra.com.br .

2 Professores Adjunto do Departamento de Tecnologia de Alimentos, UFC. Universidade Federal do Ceará - Depto. de Tecnologia de Alimentos; Avenida Mister Hull s/n - Campus do Pici - Bloco 848 - Caixa Postal 12.140 Cep 60021970 - Fortaleza - CE. E-mail: evania@ufc.br; correia@ufc.br; pblconstant@ufc.br.

* Autor para correspondência.
} 
were different on the characteristics physical-chemistry and sensorial of the tomato dry through the year storage. In this case, use from mixture $\mathrm{NaCl}$ and sucrose showed betters results concerning the acceptability. Concerning the time of storage, however indicating alterations physical-chemistry, there were no differences on the sensorial evaluation.

Key words: Drying, tomato, osmotic Dehydration

\section{Introdução}

Após a colheita, o tomate (Lycopersicon esculentum Mill) maduro apresenta em média vida de prateleira de uma semana, com perdas pós-colheita de $25-50 \%$, e, para o tomate parcialmente maduro, estes números são de 1-2 semanas, com $20-40 \%$ de perdas pós-colheita (BARRETT REINA, 1990 apud FERREIRA; FRANCO; TAVARES, 2005). Mukai e Kimura (1986) também observaram que as perdas em tomate variaram de 0 a $50 \%$ dependendo do tipo de varejo e da época do ano.

A industrialização do tomate é importante, não apenas por evitar perdas mas também por impedir uma oferta excessiva do fruto no período da safra, levando a desvalorização do produto, além de permitir agregação de valor com o fornecimento de produtos processados. Neste contexto, estudos de novas formas de processamento do tomate é interessante, e entre as diversas possibilidades, a desidratação representa uma excelente alternativa.

A desidratação osmótica, designada por desidratação-impregnação por imersão (DII) em soluções concentradas, tem por objetivo a obtenção de alimentos de umidade intermédia (AHI), seja como pré-tratamento em processos clássicos de estabilização, seja como técnica propriamente dita na obtenção de produtos estabilizados à temperatura ambiente. O processo consiste na eliminação de água do alimento, sem mudança de fase, devido à diferença de potencial osmótico que se verifica entre os produtos e a solução hipertônica desidratante (GARCIA-MUÑOZ; RIAÑO-LUNA， 1997). Como nesse processo ocorre apenas uma retirada parcial da água, resultando em um produto de baixa estabilidade a aplicação de outro processo se faz necessária, como a secagem ou o congelamento. Uma das vantagens em se utilizar à desidratação osmótica como pré-tratamento é devido às características nutricionais (vitaminas e minerais) e sensoriais (aroma, cor e textura), resultando, muitas vezes, em produtos de qualidade superior àquelas obtidas pela secagem direta.

O tomate pode ser considerado um alimento funcional devido ao alto teor de licopeno, e está presente no cardápio de restaurantes e em estabelecimentos de comidas finas. $\mathrm{O}$ tomate desidratado é produzido por pequenos e micro empresários, por métodos artesanais, mas quando se trata de médios e grandes volumes de produção, torna-se importante o desenvolvimento de um processo que, além de resultar num produto de alta qualidade, seja viável e de fácil transposição de escala (TONON; BARONI; HUBINGER, 2006).

O interesse pela desidratação osmótica como prétratamento da secagem de tomate vem aumentando consideravelmente, pois apresenta vantagens sobre outros métodos de preservação, como minimizar os danos causados pelo calor à cor, textura e sabor além do decréscimo de compostos (como licopeno e vitamina $\mathrm{C}$ ), retendo estes nutrientes durante o processamento de tomate (ALVES; SILVEIRA, 2002)

Diante do exposto, o este trabalho tem como objetivo avaliar a influência do processo de desidratação osmótica seguido de secagem por convecção forçada em secador de cabine, nas características físico-químicas, microbiológicas e sensoriais do tomate.

\section{Material e métodos}

Os tomates de mesa, variedade Santa Cruz vermelha, foram adquiridos no comércio em Fortaleza-Ce na forma in natura sendo selecionados 
quanto aos atributos de qualidade (tamanho,cor e ausência de defeitos). No laboratório, a lavagem foi feita com solução de hipoclorito de sódio a 200ppm/15minutos. Os tomates foram cortados em quatro partes, no sentido longitudinal retirando as sementes. Uma parte foi imersa em solução a $10 \%(\mathrm{p} / \mathrm{v})$ de $\mathrm{NaCl}$ e a outra em solução $5 \%(\mathrm{p} / \mathrm{v})$ de $\mathrm{NaCl}$ e $10 \%$ (p/v) de sacarose, na proporção, tomate:salmoura 1:2, por 30 minutos. Após essas etapas foram submetidos à desidratação em secador de cabine modelo Menoli PD-25 com temperatura de $65^{\circ} \mathrm{C}$, velocidade do ar em torno de $1,6 \mathrm{~m} / \mathrm{s}$ por 14h. Todas as etapas do preparo e higienização dos frutos seguiram as normas das Boas Práticas de Fabricação (BRASIL, 2002)

Foi empregado como condimento para a conserva uma mistura de azeite de oliva e óleo de girassol na proporção $1: 4$ e orégano a $0,2 \%(\mathrm{p} / \mathrm{v})$, aquecido a temperatura de $90{ }^{\circ} \mathrm{C}$ por 1 minuto em tacho aberto com camisa de vapor.

Foram acondicionados $300 \mathrm{~g}$ tomates desidratados em potes de vidro esterilizados de capacidade $500 \mathrm{~g}$. O condimento foi acrescentado quente até completar a capacidade dos frascos que foram fechados e levados para pasteurização em água fervente por 5 minutos, sendo armazenados ao abrigo de luz à temperatura ambiente de $27 \pm 2^{\circ} \mathrm{C}$ durante 45 dias para a realização das análises.

\section{Análises químicas e físico-químicas}

As análises foram realizadas com o produto innatura e após desidratado nos tempos $0,15,30$ e 45 dias de armazenamento, quanto ao teor de sólidos solúveis ( ${ }^{\circ}$ Brix), umidade (\%), atividade de água $\left(\mathrm{a}_{\mathrm{w}}\right)$, acidez titulável total $(\%), \mathrm{pH}$, açúcares totais e redutores $(\%)$, carotenóides totais $(\%)$ e cor (escala CIELab). Para cada análise 5 gramas de tomate foi triturada. A acidez titulável total foi feita segundo as normas analíticas do Instituto Adolfo Lutz (2004). O teor de sólidos solúveis foi determinado utilizando-se refratômetro da marca ATAGO N-1 com da medida dos ${ }^{\circ}$ Brix, sendo ajustado para 20
${ }^{\circ} \mathrm{C}$ e com escala variando de 0 a $32{ }^{\circ} \mathrm{Brix}$. $\mathrm{O} \mathrm{pH}$ foi determinado de acordo com a AOAC (1995) com um potenciômetro de marca WTW, modelo 330i/ SET, previamente calibrado com soluções tampão de $\mathrm{pH} 4,0$ e 7,0. Atividade de água foi medida em aparelho digital AQUALAB da marca Decagon Devices Inc. EUA modelo CX-2, com sensibilidade de 0,001 à temperatura de $\left(28^{\circ} \mathrm{C} \pm 2^{\circ} \mathrm{C}\right)$. Os açúcares redutores e totais foram determinados pelo método do ácido dinitrosalicílico (DNS), segundo Miller (1959). A leitura foi realizada em espectrofotômetro marca MICRONAL, modelo B582, utilizando-se comprimento de onda a $540 \mathrm{~nm}$. Os resultados foram expressos em percentual de glicose. Os carotenóides totais foram determinados segundo Higby (1962), sendo a extração efetuada em solução de álcool isopropílico: hexano $(3: 1)$ e a leitura efetuada em espectrofotômetro MICRONAL, modelo B582, usando o comprimento de onda a $450 \mathrm{~nm}$, expressos em miligramas de carotenóides/100 g.

Os parâmetros da cor foram determinados utilizando-se um colorímetro Minolta, modelo CR 10, obtendo-se os valores de $L^{*}, a^{*}$ e b*. L representa a luminosidade, $\mathbf{a}^{*}$ define a transição da cor verde $\left(-a^{*}\right)$ para a cor vermelha $\left(+a^{*}\right)$ e $\mathbf{b}^{*}$ representa a transição da cor azul $\left(-b^{*}\right)$ para a cor amarela $\left(+b^{*}\right)$ (SETSER, 1984).

\section{Análise sensorial}

Para as análises sensoriais foram recrutados 40 provadores não treinados de ambos os sexos, servidos monadicamente sob condições controladas. Cada provador avaliou as amostras dos dois tratamentos em uma sessão. As amostras foram servidas em pratos e acompanhadas com pão de forma para melhor avaliação do provador, codificadas com números aleatórios de três dígitos. (MACFIE et al., 1989).

Os testes de aceitação sensorial foram realizados com o produto para os atributos de aparência, cor, sabor, textura no tempo inicial 0 após 45 dias de armazenamento através da escala hedônica, 
estruturada em nove pontos, onde nove equivale à nota máxima "gostei muitíssimo" e um a nota mínima "desgostei muitíssimo" (PERYAM; PILGRIM, 1957).

Para avaliação de intenção de compra também foi utilizada escala hedônica estruturada de cinco pontos, onde cinco corresponde a "certamente compraria", a nota três "talvez comprasse, talvez não comprasse" e um a "certamente não compraria" (MEILGAARD; CIVILLE; CARR, 1987).

\section{Análises microbiológicas}

Para verificar as condições microbiológicas do produto acabado, os tomates secos foram analisados quanto à presença de Coliformes totais, Coliformes fecais, Bolores e Leveduras. A metodologia aplicada para investigação dos referidos microrganismos, seguiu ao descrito no Compendium of Methods for the Microbiological Examination of Foods, editado pela Americam Public Health Association (APHA, 2001).

\section{Delineamento experimental}

Foi utilizado delineamento experimental inteiramente casualizado com esquema fatorial $2 \times 4$ - dois tratamentos e 4 tempos de armazenamento $(0,15,30$ e 45 dias $)$ de amostragens para as análises físico-químicas e microbiológicas, realizadas em triplicatas e para a análise sensorial foi utilizado esquema fatorial $2 \times 2$ - dois tratamentos e dois tempos de armazenamento (zero e 45 dias) com 40 provadores. Os resultados foram submetidos à análise de variância (ANOVA) e comparação de médias pelo Teste de Tukey ao nível de 5\% de significância utilizando-se o software Statistica ${ }^{\circledR}$ 6.0 .

\section{Resultados e discussões}

Os resultados das análises físico-químicas foram usados para verificar a qualidade do produto final, correlacionando com os resultados da avaliação sensorial, e avaliar o efeito exercido por cada solução osmótica sobre o produto em termos de retenção ou perda de alguns constituintes. Os resultados das análises físico-químicas do produto na forma in-natura e na forma desidratada encontram-se, respectivamente, na Tabelas 1, 2 e 3 .

Comparando-se os resultados apresentados na (Tabela 1) com aqueles das (Tabelas 2 e 3 ) é possível perceber as alterações básicas promovidas pela desidratação como diminuição da umidade e, consequentemente, concentração de solutos expressos em ácidos, açúcares totais e redutores e carotenóides totais.

$\mathrm{Na}$ conserva cuja osmodesidratação foi efeita com $\mathrm{NaCl}$ e sacarose (Tratamento B) foi observado leve e gradativa redução da atividade de água ao longo do armazenamento e no Tratamento A (osmodesidratação apenas com $\mathrm{NaCL}$ ) até 30 dias, quando então não houve variação significativa. Foi demonstrado por Tonon, Baroni e Hubinger (2006), Antonio et al. (2006) e Azoubel e Murr (2004) que a presença do $\mathrm{NaCl}$ provoca uma redução da atividade de água da solução, aumentando, assim, a força motriz que provoca a saída de água do produto. No presente experimento, os valores de atividade de água foram mais baixos para o tratamento $\mathrm{B}$, uma vez que tal tratamento a desidratação osmótica anterior a secagem foi efetuada com sacarose além do $\mathrm{NaCl}$. Nesse caso a pressão osmótica mais acentuada promoveu uma maior desidratação que é comprovada pelos resultados do teor de umidade inicial menor. A tendência de decréscimo de umidade continuou para ambos os tratamentos, indicando que houve perda de água do produto para o meio durante $o$ armazenamento. 
Tabela 1. Resultados das análises físico-químicas do tomate de mesa, variedade Santa Cruz vermelha, na forma innatura.

\begin{tabular}{cccccccc}
\hline $\mathbf{a}_{\mathbf{w}}$ & $\begin{array}{c}\text { Umidade } \\
(\mathbf{\%})\end{array}$ & $\begin{array}{c}\mathbf{S S T}^{\mathbf{1}} \\
\left({ }^{\circ} \mathbf{B r i x}\right)\end{array}$ & $\begin{array}{c}\text { ATT } \\
(\mathbf{\%})\end{array}$ & $\mathbf{p H}$ & $\begin{array}{c}\mathbf{A T}^{3} \\
(\mathbf{\%})\end{array}$ & $\begin{array}{c}\mathbf{A R}^{4} \\
(\mathbf{\%})\end{array}$ & $\begin{array}{c}\mathbf{C T}^{\mathbf{5}} \\
(\mathbf{m g} / \mathbf{1 0 0 g})\end{array}$ \\
\hline 0,99 & 94,4539 & 5,00 & 0,28 & 4,41 & 2,84 & 2,67 & 1,36 \\
\hline
\end{tabular}

${ }^{1}$ Sólidos totais, ${ }^{2}$ Acidez titulável total, ${ }^{3}$ Açúcares totais, ${ }^{4}$ Açúcares redutores e ${ }^{5}$ Carotenóides totais

Tabela 2. Valores de atividade de água, umidade, sólidos totais, acidez total de tomates desidratados nos tratamentos A (salgado) e B (doce) durante 45 dias de armazenamento.

\begin{tabular}{|c|c|c|c|c|c|c|c|c|}
\hline \multirow{2}{*}{$\begin{array}{l}\text { Tempo } \\
\text { (dias) }\end{array}$} & \multicolumn{2}{|c|}{$a_{w}$} & \multicolumn{2}{|c|}{ Umidade (\%) } & \multicolumn{2}{|c|}{$\begin{array}{l}\text { Sólidos solúveis } \\
\text { totais }\left({ }^{\circ} \text { Brix) }\right.\end{array}$} & \multicolumn{2}{|c|}{$\begin{array}{l}\text { Acidez Titulável total } \\
\text { (\% ácido cítrico) }\end{array}$} \\
\hline & A & B & A & B & A & $\mathrm{B}$ & A & B \\
\hline 0 & $0,933 \mathrm{a}$ & $0,945 \mathrm{a}$ & $67,9363 \mathrm{a}$ & $62,8020 \mathrm{a}$ & $24,33 \mathrm{~b}$ & $28,33 \mathrm{c}$ & $1,22 \mathrm{~b}$ & $1,19 \mathrm{~b}$ \\
\hline 15 & $0,926 \mathrm{~b}$ & $0,930 \mathrm{~b}$ & $65,9784 a b$ & $59,2109 \mathrm{ab}$ & $25,47 \mathrm{~b}$ & $28,70 \mathrm{c}$ & $1,51 \mathrm{a}$ & $1,49 \mathrm{a}$ \\
\hline 30 & $0,918 \mathrm{c}$ & $0,894 \mathrm{c}$ & $61,8032 \mathrm{ab}$ & $55,0561 \mathrm{~b}$ & $31,67 \mathrm{a}$ & $38,00 \mathrm{~b}$ & $1,01 \mathrm{c}$ & $1,12 \mathrm{~b}$ \\
\hline 45 & $0,914 \mathrm{c}$ & $0,886 \mathrm{~d}$ & $55,1472 \mathrm{~b}$ & $53,4685 \mathrm{~b}$ & $33,33 \mathrm{a}$ & $42,53 a$ & $0,89 \mathrm{~d}$ & $0,94 \mathrm{c}$ \\
\hline
\end{tabular}

*Médias seguidas de pelo menos uma mesma letra na mesma coluna, não diferem ao nível de 5\% pelo teste Tukey.

Tais resultados são acompanhados de um aumento no teor de sólidos totais (tabela 2). As amostras de tomate in natura apresentaram teor médio de sólidos solúveis totais (SST) de $5,0^{\circ}$ Brix, que foi aumentado em média para 24,33 no tratamento A e 28,33 no tratamento $\mathrm{B}$, após a desidratação osmótica e secagem. Este efeito no teor de SST foi observado por Antonio et al. (2006), que encontraram valores de SST semelhantes aos do presente estudo, onde resultou que em soluções com $10 \%$ de sal e altas concentrações de sacarose, o ganho de sólidos tende a ser menor que em soluções contendo apenas sacarose.

Os valores obtidos para acidez titulável total (ATT) das amostras diferiram entre si, sendo observado um ligeiro aumento entre os tempos 0 e 15 dias, seguido de decréscimo até o tempo de 45 dias. O aparente aumento inicial foi observado pela maior concentração devido a perda de umidade ao longo do tempo de armazenamento. No entanto ocorre a oxidação dos ácidos orgânicos, principalmente se a secagem for precedida por uma desidratação osmótica (ALVES; SILVEIRA, 2002). Esses resultados estão de acordo com os apresentados por Maestrelli et al. (2001), que observaram redução da acidez titulável de 19,17 e 21,14 para 11,84 e 11,07 meq 100 g-1 $(38,24$ e $47,63 \%$ ) respectivamente, em esferas de melão desidratadas em solução de sacarose a $60 \%$. Peiró et al. (2006) verificaram perdas de ácido cítrico entre 18 e $32 \%$ e entre 30 e $44 \%$, em operações sucessivas de desidratação osmótica de "grapefruit" (toranja) e abacaxi, respectivamente, em soluções de sacarose a $55^{\circ}$ Brix. A tendência geral de redução da acidez ao longo do tempo pode ser atribuída a oxidações dos ácidos orgânicos e foi observada por outros autores em estudos semelhantes com diferentes produtos de origem vegetal (CARVALHO; GUERRA, 1995; FREITAS et al., 2006). 
Tabela 3. Valores de pH, açúcar total e redutor e carotenóides de tomates desidratados nos tratamentos A (salgado) e B (doce) durante 45 dias de armazenamento.

\begin{tabular}{ccccccccc}
\hline \multirow{2}{*}{ Tempo (dias) } & \multicolumn{2}{c}{$\mathbf{p H}$} & \multicolumn{2}{c}{ Açúcar total (\%) } & \multicolumn{2}{c}{ Açúcar redutor (\%) } & \multicolumn{2}{c}{$\begin{array}{c}\text { Carotenóide total } \\
\text { (mg/100g) }\end{array}$} \\
\cline { 2 - 9 } & $\mathrm{A}$ & $\mathrm{B}$ & $\mathrm{A}$ & $\mathrm{B}$ & $\mathrm{A}$ & $\mathrm{B}$ & $\mathrm{A}$ & $\mathrm{B}$ \\
\hline 0 & $4,05 \mathrm{~d}$ & $4,13 \mathrm{c}$ & $9,82 \mathrm{c}$ & $15,40 \mathrm{~d}$ & $9,12 \mathrm{~d}$ & $14,79 \mathrm{~d}$ & $1,72 \mathrm{a}$ & $2,09 \mathrm{a}$ \\
15 & $4,09 \mathrm{c}$ & $4,22 \mathrm{~b}$ & $26,01 \mathrm{a}$ & $19,61 \mathrm{c}$ & $12,72 \mathrm{c}$ & $19,36 \mathrm{c}$ & $1,65 \mathrm{ab}$ & $1,89 \mathrm{a}$ \\
30 & $4,21 \mathrm{~b}$ & $4,20 \mathrm{~b}$ & $20,89 \mathrm{~b}$ & $31,61 \mathrm{~b}$ & $13,99 \mathrm{~b}$ & $23,81 \mathrm{~b}$ & $1,62 \mathrm{ab}$ & $1,58 \mathrm{~b}$ \\
45 & $4,29 \mathrm{~d}$ & $4,26 \mathrm{a}$ & $17,56 \mathrm{~b}$ & $44,11 \mathrm{a}$ & $16,73 \mathrm{a}$ & $30,45 \mathrm{a}$ & $1,50 \mathrm{~b}$ & $1,49 \mathrm{~b}$ \\
\hline
\end{tabular}

*Médias seguidas de pelo menos uma mesma letra na mesma coluna, não diferem ao nível de 5\% pelo teste Tukey.

$\mathrm{O} \mathrm{pH}$ apresentou aumento leve, porém significativo ao nível de $5 \%$ de probabilidade pelo teste Tukey, compatível com os resultados de diminuição da acidez e foram semelhantes aos obtidos por Queiroz et al. (2007) ao estudar a desidratação e secagem de goiaba. $\mathrm{O}$ pH influencia no sabor do produto, sendo desejável, nesse produto, valor inferior a 4,5 .

Os teores de açúcares totais apresentaram aumento significativo durante o armazenamento para ambos os tratamentos, o que é justificado pela diminuição da umidade. Para os teores de açúcares redutores foram observadas alterações com o tempo de armazenamento, que apresentaram significativo aumento em ambos os tratamentos. Em termos percentuais houve aumentos na ordem de $83,44 \%$ para o tratamento A e $105,88 \%$ para o tratamento B. Tais resultados são produto da hidrólise gradativa dos açúcares não redutores pela presença de ácido e levam a alterações sensoriais uma vez que os açúcares redutores apresentam um poder de doçura maior.

Os carotenóides totais encontrados nos tomates desidratados variaram com o tempo de armazenamento de 1,72 a $1,50 \mathrm{mg} / 100 \mathrm{~g}$ no tratamento A e de a 2,09 a $1,49 \mathrm{mg} / 100 \mathrm{~g}$ no tratamento B. Uma vez que o licopeno é o principal carotenóide encontrado no tomate (WILBERG; RODRIGUEZ-AMAYA, 1993) uma queda no seu teor acarretará em uma perda da coloração avermelhada que é constatado pela (Figura 1) pela diminuição da coordenada colorimétrica $\mathrm{a}^{*}$.

A (Tabela 4) mostra os resultados colorimétricos para o tomate in natura e logos após a desidratação para ambos os tratamentos.

Tabela 4. Coordenadas colorimétricas do tomate na forma in natura e desidratado.

\begin{tabular}{|c|c|c|c|c|c|c|c|c|}
\hline Produto & \multicolumn{2}{|c|}{$\mathbf{L}^{*}$} & \multicolumn{2}{|c|}{$a^{*}$} & \multicolumn{2}{|c|}{$\mathbf{b}^{*}$} & \multicolumn{2}{|c|}{$c^{*}$} \\
\hline In natura & \multicolumn{2}{|c|}{31,96} & \multicolumn{2}{|c|}{19,58} & \multicolumn{2}{|c|}{8,90} & \multicolumn{2}{|c|}{21,51} \\
\hline & $\mathrm{A}$ & $\mathrm{B}$ & $\mathrm{A}$ & $\mathrm{B}$ & $\mathrm{A}$ & $\mathrm{B}$ & $\mathrm{A}$ & $\mathrm{B}$ \\
\hline Desidratado & 33,02 & 31,55 & 27,24 & 25,96 & 23,67 & 18,84 & 36,09 & 32,08 \\
\hline
\end{tabular}


Foi observado um aumento da coordenada L no produto desidratado no tratamento A e efeito contrário para o tratamento $\mathrm{B}$. De acordo com Queiroz et al. (2007) esse fato pode ter ocorrido em razão da natureza do soluto impregnado no fruto, que associado ao aumento da temperatura, durante a secagem, favoreceu os processos de escurecimento não enzimático, como as reações de Maillard, caramelização e oxidação do ácido ascórbico. Sousa et al. (2003) verificou, em estudos com bananas desidratadas por pré-tratamento osmótico (a pressão atmosférica e a vácuo) seguido de secagem em estufa, diminuição no valor de $\mathrm{L}^{*}$ quando se compara o produto in natura com o desidratado e no decorrer do tempo de estocagem dos produtos, à temperatura ambiente.

As demais coordenadas colorimétricas apresentaram tendência de aumento para ambos os tratamentos principalmente devido ao efeito da concentração pelo processo de secagem. Tal efeito é facilmente percebido quando se analisa a coordenada $\mathrm{c}^{*}$ que determina a saturação da cor.

A (Figura 1) apresenta os valores médios da luminosidade $\left(\mathrm{L}^{*}\right)$, intensidade de vermelho $\left(\mathrm{a}^{*}\right)$ e intensidade de amarelo $\left(b^{*}\right)$ das amostras de tomate desidratados, nos diferentes tempos de armazenamento.

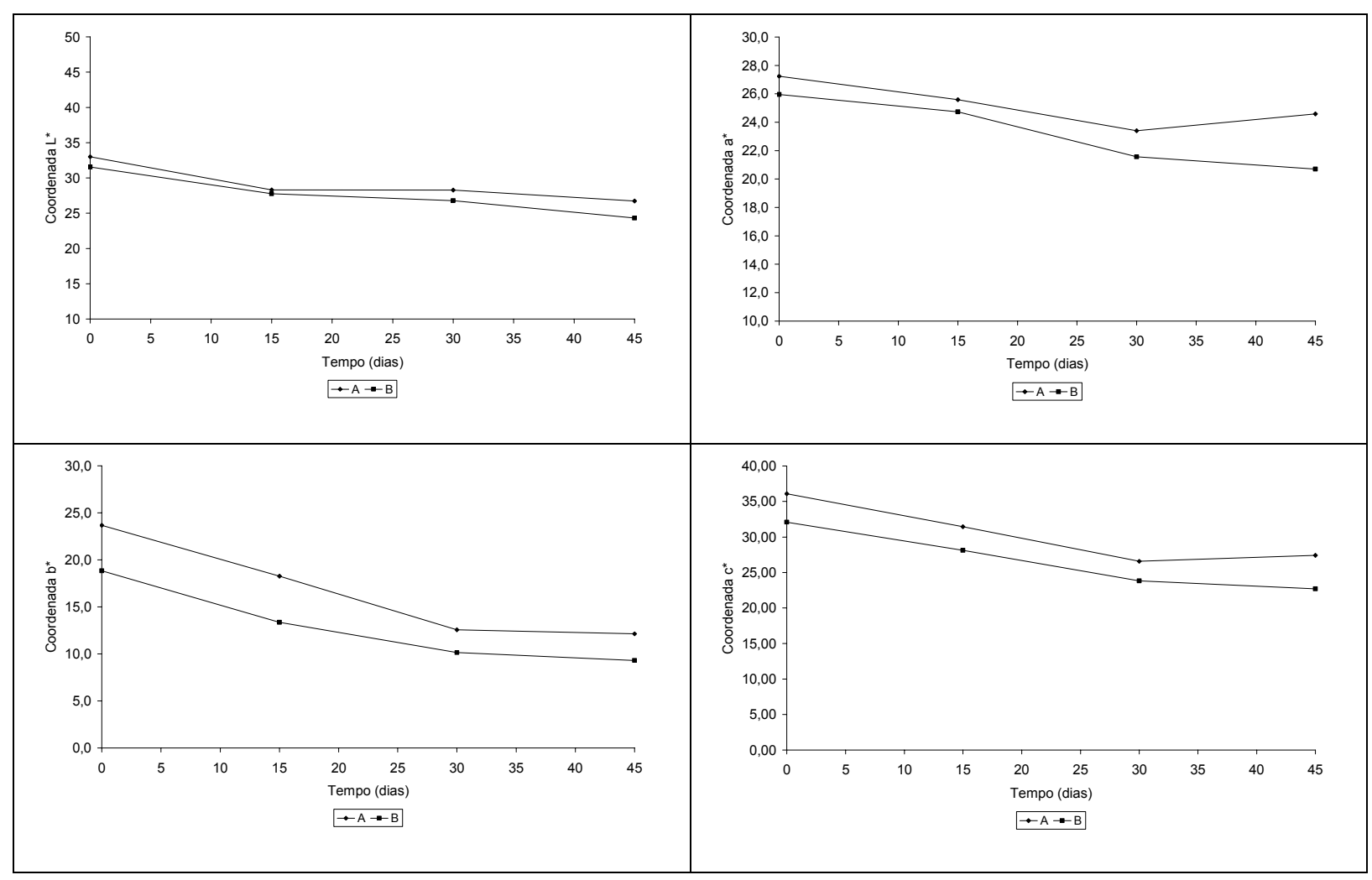

Figura 1. Médias para as coordenadas colorimétricas $\mathrm{L}^{*}, \mathrm{a}^{*}, \mathrm{~b}^{*} \mathrm{e} \mathrm{c}^{*}$ de tomates desidratados nos tratamentos A (Salgado) e B (Doce) durante o armazenamento. 
Foram observados, ao longo do armazenamento, decréscimos na luminosidade (redução do valor $L^{*}$ ) em ambas as amostras devido ao escurecimento não enzimático.

Com relação à coordenada $\mathrm{a}^{*}$ houve um decréscimo para os tratamentos A e B sendo este último mais acentuado. Uma vez que o valor $\mathrm{a}^{*}$ quantifica a intensidade de vermelho, este fenômeno era esperado, visto que em processo de degradação os componentes vermelho/alaranjados que constituem os pigmentos carotenóides transformam-se em um grupo de compostos amarelos (McCKEOWN; MARK, 1962; SCOTTER et al., 1994). Portanto, o valor de $\mathrm{a}^{*}$ deve diminuir com o tempo se estiver ocorrendo alguma degradação do produto avaliado. Quando se compara os resultados colorimétricos com a quantificação do teor de carotenóides totais mostrados na Tabela 2, verifica-se que de fato a amostra B apresentou uma queda mais acentuada, indicando uma maior perda desses pigmentos.

A avaliação da coordenada $b^{*}$ mostra comportamento semelhante de queda para as duas formulações, ou seja, diminuição dos componentes amarelos. Deve-se considerar que ao mesmo tempo que compostos vermelhos estão sendo degradados, os amarelos também estão. Isto ocorre em taxas de variação distintas. De fato, segundo Scotter (1995), as formas amarelas seriam mais estáveis que os compostos de cor vermelha nos carotenóides.

O decréscimo geral dos pigmentos leva a uma perda da saturação da cor conforme foi observado pela diminuição da coordenada c*.

Resultados semelhantes foram verificados por Queiroz et al. (2007), que encontraram redução dos valores de L*,a* eb* após 30 dias de armazenamento de goiabas desidratadas e secas. Pereira, Queiroz e Figueiredo (2006) observaram redução do valor de b* após 60 dias de armazenamento de tomate em pó.

\section{Análises microbiológicas}

Os tomates obtidos segundo os tratamentos A e B logo após a desidratação no secador de cabine (tempo 0) e durante os 45 dias de armazenamento, apresentaram contagens de bactérias mesofílicas, bolores e leveduras inferiores a $10 \mathrm{UFC} / \mathrm{mL}$, valores de coliformes totais inferiores a $3 \mathrm{NMP} / \mathrm{g}$. Os produtos atenderam aos padrões estabelecidos pela legislação vigente (BRASIL, 2001)

\section{Análise sensorial}

Os atributos sensoriais de cor, sabor, aparência e intenção de compra não diferiram ao longo do tempo de armazenamento pelo teste $\mathrm{F}$ na análise de variância ao nível de 5\% de probabilidade. Porém no atributo textura foi observada diferença significativa com o tempo de armazenamento. As médias dos atributos sensoriais estão apresentadas na (Tabela 5) e os resultados da Anova para o atributo textura dos tomates desidratados estão apresentados no (Quadro 1).

Tabela 5. Médias das notas atribuídas pelos provadores à cor,aparência,sabor,textura e intenção de compra de tomate seco.

\begin{tabular}{ccccccccccc}
\hline \multirow{2}{*}{ Tempo (dias) } & \multirow{2}{*}{ Cor } & \multicolumn{2}{c}{ Aparência } & \multicolumn{2}{c}{ Sabor } & \multicolumn{2}{c}{ Textura } & \multicolumn{2}{c}{$\begin{array}{c}\text { Intenção de } \\
\text { compra }\end{array}$} \\
\hline & A & B & A & B & A & B & A & B & A & B \\
\hline 0 & 7,2 & 7,1 & 6,7 & 6,6 & 7,4 & 7,7 & 6,9 & 7,6 & 3,7 & 3,9 \\
45 & 7,3 & 7,1 & 7,1 & 6,8 & 7,1 & 7,5 & 6,9 & 7,5 & 3,8 & 4,0 \\
\hline
\end{tabular}


Quadro 1. Análise de variância dos dados do atributo textura do tomate para o tempo 0 .

\begin{tabular}{lccccc}
\hline Fontes de variação & GL & SQ & QM & Fc & \\
\hline Amostra ( A ) & 1 & 7,8125 & 7,8125 & 7,677165 & $*$ \\
\hline Julgadores ( J ) & 39 & 138,9875 & 3,563782 & & \\
\hline Resíduo ( R ) & 39 & 39,6875 & 1,017628 & & \\
\hline Total & 79 & 186,4875 & & & \\
\hline
\end{tabular}

* significativo ao nível de 5\% de probabilidade

Quadro 2. Análise de variância dos dados do atributo textura do tomate para o tempo 45

\begin{tabular}{lccccl}
\hline Fontes de variação & GL & SQ & QM & Fcalc & \\
\hline Amostra ( B ) & 1 & 7,2 & 7,2 & 5,420849 & $*$ \\
\hline Julgadores ( J ) & 39 & 72,95 & 1,870513 & & \\
\hline Resíduo ( R ) & 39 & 51,8 & 1,328205 & & \\
\hline Total & 79 & 131,95 & & & \\
\hline
\end{tabular}

* significativo ao nível de $5 \%$ de probabilidade

Embora não tenha sido determinadas diferenças significativas, as amostras do tratamento A receberam melhores notas quanto às características de cor e aparência tanto no tempo inicial como após 45 dias de armazenamento. Isto pode ser explicado pelo fato de que tomates secos contendo maior grau de umidade apresentam uma aparência mais atraente comparada à dos produtos com menor umidade, devido principalmente à característica de enrugamento superficial, menos acentuada para os produtos menos secos. Camargo, Haj-Isa e Queiroz (2007) obteve notas entre 3,8 e 4,0 para cor, e entre 3,3 e 3,7 para aparência, sendo inferiores às observadas no presente estudo para ambos os tratamentos.

Em relação ao atributo sensorial de sabor, os provadores atribuíram maiores notas ao tratamento B (7,7 que corresponde entre gostei moderadamente e gostei muito) quando comparadas ao tratamento $\mathrm{A}$ $(7,1)$. De acordo com Venske et al. (2004), isto pode ser atribuído aos valores elevados de SST e pH dos tomates do tratamento B.

Para a textura foi observada diferença significativa entre os tratamentos tanto na degustação no tempo 0 quanto no tempo 45 dias (Quadro 2). Os tomates do tratamento $\mathrm{B}$ receberam em média nota maior que os tomates do tratamento A, indicando a preferência para o tratamento B quanto ao atributo textura. A diferença pode ser atribuída ao menor teor de umidade das amostras do tratamento B em relação ao tratamento A, garantindo a firmeza típica de produtos secos, sendo conseqüentemente mais apreciados pelos provadores. Com o tempo de armazenamento, os tomates de ambos os tratamentos receberam nota 7 (gostei moderadamente) em média.

Com relação à intenção de compra dos tomates, tanto no tempo inicial quanto no final da análise, ambos os tratamentos apresentaram nota média abaixo de 5,0 (limite inferior de aceitação), conforme a escala hedônica de 9 pontos utilizada nos testes de aceitação e intenção de compra. Contudo, as notas em média para intenção de compra nos dois tratamentos ficou entre 3 e 4 o que corresponde na escala hedônica a possivelmente compraria. Este resultado pode ser atribuído à baixa freqüência de consumo de tomate seco pelos provadores.

De uma forma geral não houve diferenças marcantes de aceitação durante o armazenamento, que é interessante uma vez que o produto pode ser consumido logo após a fabricação e tendo um tempo de prateleira longo. 


\section{Conclusões}

Foi possível constatar que o emprego de desidratação osmótica, partindo-se de solutos e concentrações distintas, influenciou de forma diferenciada nas características físicoquímicas e sensoriais do tomate seco ao longo do armazenamento.

No presente caso o emprego da mistura $\mathrm{NaCl}$ e sacarose levou a melhores resultados no que diz respeito a aceitação.

Com relação ao tempo de armazenamento, não foi constatada diferenças nas avaliações sensoriais, indicando que embora tenham ocorrido alterações físico-químicas, elas não foram significativamente percebidas pelos provadores.

\section{Referências}

ALVES, S. M.; SILVEIRA, A. M. Estudo da secagem de tomates desidratados e não desidratados osmoticamente. Revista Universidade Rural Série Ciências Exatas e da Terra, Seropedica, v. 21, n. 1, p. 21-30, 2002.

ANTONIO, G. C.; KUROSAWA, L. E.; MURR, F. E. X.; PARK, K. J. Otimização da desidratação osmótica de batata doce (Ipomoea batatas) utilizando metodologia de superfície de resposta. Brazilian Journal of Food Technology, Campinas, v. 9, n. 2, p. 135-141, abr.jun. 2006.

ASSOCIATION OF OFFICIAL ANALYTICAL AOAC. Official methods of analysis of the association of official analytical chemistry. 16. ed. Washington: AOAC, 1995.

AMERICAN PUBLIC HEALTH ASSOCIATION APHA. Compendium of methods for the microbiological examination of foods. Washington: APHA, 2001.

AZOUBEL, P. M.; MURR, F. E. X. Mass transfer kinetics of osmotic dehydration of cherry tomato. Journal of Food Engineering, Essex, v. 61, n. 3, p. 291-295, 2004.

BRASIL. Ministério da Saúde. Agência Nacional de Vigilância Sanitária. Resolução RDC, $n^{\circ}$ de 12, de 02 de janeiro de 2001. Dispõe sobre os princípios gerais para o estabelecimento de critérios e padrões microbiológicos para alimentos. Disponível em: <www.anvisa.gov.br>. Acesso em: 08 jan. 2005.
BRASIL. Ministério da Saúde. Agência Nacional de Vigilância Sanitária. Resolução RDC, $n^{\circ}$ de 352, de 23 de dezembro de 2002. Regulamento Técnico de Boas Práticas de Fabricação para Estabelecimentos Produtores/ Industrializadores de Frutas e ou Hortaliças em Conserva. Disponível em: <www.anvisa.gov.br>. Acesso em: 08 jan. 2005.

CAMARGO, G. A.; HAJ-ISA, N.; QUEIROZ, M. R. Avaliação da qualidade de tomate seco em conserva. Revista Brasileira de Engenharia Agrícola e Ambiental, Campina Grande, v. 11, n. 5, p. 521-526, 2007.

CARVALHO, I. T.; GUERRA, N. B. Suco de acerola estabilidade durante o armazenamento. In: SÃO JOSÉ, A. R.; ALVES, R. E. Cultura de Acerola no Brasil: produção de mercado. Vitória da Conquista: DFZ/UESB, 1995. p. 102-105.

FERREIRA, M. D.; FRANCO, A. T. O.; TAVARES, M. Técnicas de colheita para tomate de mesa. Horticultura Brasileira, Brasília, v. 23, n. 4, p. 1018-1021, out/dez. 2005.

FREITAS, C. A. S.; MAIA, G. A.; COSTA, J. M. C.; FIGUEIREDO, R. W.; RODRIGUES, M. C. P.; SOUZA, P. H. M. Estabilidade do suco de acerola (Malpighia amarginata D.C.) adoçado envasado pelos processos Hot-fill e asséptico. Ciência e Tecnologia de Alimentos, Campinas, v. 26, n. 3, p. 544-549, jul./set. 2006.

GARCIA-MUÑOZ; RIAÑO-LUNA. Influencia de algunas variables sobre el proceso de deshidratación osmótica de mango, banano y aguacate. Cenicafé, Colombia, v. 48, n. 2, p.109-119. 1997

HIGBY, W. K. A simplified method for determination of some the carotenoid distribution in natural and carotenefortified orange juice. Journal of Food Science, Chicago, v. 27 , n. 1, p. 42-49, 1962.

INSTITUTO ADOLFO LUTZ. Métodos físico-químicos para análise de alimentos. 4.ed. São Paulo, 2004. 1004 p.

MACFIE, H. J.; BRATCHELL, N.; GREENHOFF, K.; VALLIS, L. V. Designs to balance the effect of order of presentation and first-order carry-over effects in hall tests. Journal of Sensory Studies, Westport, v. 4, n. 2, p. 129-148, 1989.

McKEOWN, G. G., MARK, E. The composition of oilsoluble annatto food colors. Journal of the A.O.A.C., Catherine Wattenberg, v. 45, n. 3, p. 761-766, 1962.

MAESTRELLI, A.; LO SCALZO, R.; LUPI，D.; BERTOLO, G.; TORREGGIANI, D. Partial removal of water before freezing: cultivar and pre-treatments as 
quality factors of frozen muskmelon (Cucumis melo, cv. reticulatus Naud.). Journal of Food Engineering, Essex, v. 49, n. 2/3, p. 255-260, 2001.

MEILGAARD, M.; CIVILle, G. V.; CARR, B. T. Sensory Evaluation Techniques. Boca Raton: CRC Press, 1987. $2 \mathrm{v}$.

MILLER, G. L. Use of dinitrosalicilic acid reagent for determination of reducing sugar. Analytical Biochemistry, New York, v. 31, n. 3, p. 426-428, 1959.

MUKAI, M. K.; KIMURA, S. Investigação das práticas pós-colheita e desenvolvimento de um método para análise de perdas de produtos hortícolas. Viçosa: CENTREINAR, 1986.

PEIRÓ, R.; DIAS, V. M. C.; CAMACHO, M. M.; MARTÍNEZ-NAVARRETE, N. Micronutrient flow to the osmotic solution during grapefruit osmotic dehydration. Journal of Food Engineering, Essex, v. 74, n. 3, p. 299307, 2006.

PEREIRA, I. E.; QUEIROZ, A. J. M.; FIGUEIRÊDO, R. M. F. Características físico-químicas do tomate em pó durante o armazenamento. Revista de Biologia e Ciências da Terra, João Pessoa, v. 6, n. 1, p. 83-90, 2006.

PERYAM, D. R.; PILGRIM, P. J. Hedonic scale method for measuring food preferences. Food Technology, Chicago, v. 11, n. 9, p. 9-14, 1957.

QUEIROZ, V. A. V.; BERBET, P. A.; MOLINA, M. A. B.; GRAVINA, G. A.; QUEIROZ, R. L.; DELIZA, R. Desidratação por imersão-impregnação e secagem por convecção de goiaba. Pesquisa agropecuária brasileira, Brasília, v. 42, n. 10, p. 1479-1486, 2007.

SCOTTER, M. J.; THORPE, S. A.; REYNOLDS, S. L.; WILSON, L. A.; STRUTT, P. R. Characterization of the principal colouring components of annatto using higt performance liquid chromatography with photodiodearray detectio. Food Additives and Contaminants, London, v. 11, n. 3, p. 301-315, 1994.

SCOTTER, M. J. Characteritation of the coloured thermal degradation products of bixin from annatto and a reveised mechanism for their formation. Food Chemistry, London, v. 53, n. 2, p. 177-185, 1995.

SOUSA, P. H. M. DE; MAIA, G. A.; SOUZA FILHO, M. M. DE; FIGUEIREDO, R. W.; NASSU, R. T.; BORGES, M. F. Avaliação de produtos obtidos pela desidratação osmótica de banana seguido de secagem. Boletim do Centro de Pesquisa e Processamento de Alimentos, Curitiba, v. 21, n. 1, p. 109-120, 2003.

SETSER, C. S. Color: reflections and transmissions. Journal of Food Quality, Wastport, v. 6, n. 3, p. 183-197, 1984.

TONON, R. V.; BARONI, A. F.; HUBINGER, M. D. Estudo da Desidratação Osmótica de Tomate em Soluções Ternárias pela Metodologia de Superfície de Resposta. Ciência Tecnologia de Alimentos, Campinas, v. 26, n. 3, p. 715-723, jul./set. 2006.

VENSKE, C.; SANTOS, J.; RAUPP, D. S.; GARDINGO, J. R.; BORSATO, A. V. Influência do grau de maturação nas características sensoriais de tomate seco envasado em óleo. Ciências Exatas da Terra, Ponta Grossa, v. 10, n. 3, p. 33-40, 2004.

WILBERG, V. C.; RODRIGUEZ-AMAYA, D. B. Quantificação de beta-caroteno e licopeno em tomate e em alguns dos seus produtos por CLAE. Ciência e Tecnologia de Alimentos, Campinas, v. 13, p. 132-141, 1993. 
